\title{
Phase Equilibria Characteristics at and near Cloud Point of Quasi-Binary System Consisting of Multicomponent Polymers in a Single Solvent
}

\author{
Shigenobu MATSUDA \\ Fundamental Research Laboratory of Fibers and Fiber-Forming Polymers, \\ Asahi Chemical Industry Co., Ltd., \\ 11-7, Hacchonawate, Takatsuki, Osaka 569, Japan
}

(Received May 15, 1986)

\begin{abstract}
Incipient phase, isolated at cloud point from quasi-binary solution consisting of multicomponent polymers in a single solvent, was named "cloud particle" and an attempt was made to clarify the concentration and the composition (i.e., the molecular weight distribution) of the polymer in the cloud particle as a function of initial polymer volume fraction $v_{p}^{0}$. For this purpose, a theoretical method for direct calculation of the cloud point curve was employed considering concentration- and molecular weight-dependences of the polymer-solvent interaction parameter $\chi$. In order to compare characteristics (the concentration and composition of polymer) of two-phase equilibrium for $\rho_{p}>0\left(\rho_{p}\right.$, the relative amount of polymer partitioned in a polymer-rich phase) with those of the cloud particle (two-phase equilibrium for $\rho_{p}=0$ ), two-phase equilibrium for $\rho_{p}>0$ was calculated under the same conditions as those of cloud particle. Computer experiments of the cloud particle were also carried out in the range of $X_{w}^{0} / X_{n}^{0}=1.1-4, X_{w}^{0}=$ $3 \times 10^{2}-5 \times 10^{3}\left(X_{w}^{0}\right.$ and $X_{n}^{0}$ are number- and weight-average of the molar volume ratio of polymer to solvent of original polymer, respectively), $p_{1}=0-0.8$, and $p_{2}=0-0.4$ ( $p_{1}$ and $p_{2}, 1$ st and 2nd order concentration dependent parameters). With a decrease in $X_{w}^{0}$ and an increase in $p_{1}$ and $p_{2}$, polymer concentration of the cloud particle increases.

KEY WORDS Quasi-Binary Polymer Solution / Cloud Point / Cloud Particle / Molecular Weight / Molecular Weight Distribution / Concentration Dependence / $\chi$ Parameter / Polymer Concentration / Critical Solution Point /
\end{abstract}

During these fifteen years, Kamide and his coworkers ${ }^{1-8}$ and Koningsveld et al..$^{9-13}$ have clarified, based on the modified FloryHuggins ${ }^{14}$ thermodynamic theory, the effect of molecular characteristics of the original polymer, solvent nature and operating conditions on the coexisting curve and the characteristics of two co-existing phases for multicomponent polymers/single solvent system (quasi-binary system). They showed that the concentration- and molecular weight-dependences of the polymer-solvent interaction parameter $\chi$ should be considered very carefully in phase equilibrium. As an extension of their theory, Kamide et al. proposed a method for directly calculating cloud point curve (CPC) and critical solution point (CSP) of quasi-binary solutions. ${ }^{15}$ Using concentration-independent and -dependent $\chi$ parameters, Šolc ${ }^{16-22}$ suggested a direct method to calculate CPC and studied multiple critical points and three phase equilibria. But he did not study molecular characteristics of polymer in the cloud particle systematically.

Recent experiments (for example, see Figure 8 of ref 23) show that a phase separated near the cloud point is in a particle form. In this paper; incident phase, isolated at cloud 
point from quasi-binary solutions, was named the cloud particle and an attempt was made to clarify the composition and molecular characteristics of the polymer in the cloud particle. Comparisons of the cloud particle characteristics with two-phase equilibrium characteristics $\left(\rho_{p}=0.005-0.995, \rho_{p}\right.$, weight ratio of polymer partitioned in polymer-rich phase to the original polymer $)^{1-8}$ were also made. Further, the effects of the molecular weight distribution (MWD) and the weight-average molecular weight- and concentration-dependences of $\chi$-parameter on the composition of the cloud particle and molecular characteristics of polymer in the cloud particle were also studied.

\section{THEORETICAL BACKGROUND}

When the polymer volume fraction of the starting solution $v_{p}^{0}$ is lower than the critical polymer volume fraction $v_{p}^{c}$, (i.e., $\left.v_{p}^{0}<v_{p}^{c}\right)$, the two phase volume ratio $R\left(\equiv V_{(1)} / V_{(2)} ; V_{(1)}\right.$ and $V_{(2)}$ are the volume of polymer-lean and -rich phases, respectively) becomes infinite at cloud point (CP) and the volume fractions of the $X_{i}$-mer (in the strict sense, $X_{i}$ is the molar volume ratio of polymer to solvent) in polymer-lean and -rich phases, $v_{X_{i}(1)}$ and $v_{X_{i}(2)}$, at CP (referred to as $v_{X_{i}(1)}^{c p}$ and $v_{X_{i}(2)}^{c p}$, respectively) are given by eq 1 and $2,{ }^{15}$

$$
\begin{aligned}
& v_{X_{i}(1)}^{c p}=v_{X_{i}}^{0} \\
& v_{X_{i}(2)}^{c p}=v_{X_{i}}^{0} \exp \left(\sigma_{i}^{c p} X_{i}\right)
\end{aligned}
$$

where $v_{X_{i}}^{0}$ is the volume fraction of the $X_{i}$ mer of the starting solution and $\sigma_{i}^{c p}$ is the partition coefficient $\sigma_{i}$ at $\mathrm{CP}$. When $v_{p}^{0}$ is higher than $v_{p}^{c}$ (i.e., $v_{p}^{0}>v_{p}^{c}$ ), $R$ becomes zero and $v_{X_{i}(1)}^{c p}$ and $v_{X_{i}(2)}^{c p}$ are given as follows: ${ }^{15}$

$$
\begin{aligned}
& v_{X_{i}(1)}^{c p}=v_{X_{i}}^{0} \exp \left(-\sigma_{i}^{c p} X_{i}\right) \\
& v_{X_{i}(2)}^{c p}=v_{X_{i}}^{0}
\end{aligned}
$$

The cloud particles are a polymer-rich phase itself for $v_{p}^{0}<v_{p}^{c}$ and a polymer-lean phase for $v_{p}^{0}>v_{p}^{c}$. Strictly speaking, the cloud par- ticles do not have the volume $\left(V_{(2)} \rightarrow 0\right.$ for $v_{p}^{0}<v_{p}^{c}$ and $V_{(1)} \rightarrow 0$ for $\left.v_{p}^{0}>v_{p}^{c}\right)$ and accordingly they do not contain the polymer $\left(\rho_{p} \rightarrow 0\right.$ for $v_{p}^{0}<v_{p}^{c}$ and $\rho_{s} \rightarrow 0$ for $v_{p}^{0}>v_{p}^{c}, \rho_{s}$ $\left(=1-\rho_{p}\right)$, the weight ratio of polymer partitioned in polymer-lean phase to the original), but the volume fraction of polymer in the cloud particle can be defined by eq $1-4$. The cloud particle is expected to have the close correlation with primary particles first observed by Kamide and Manabe in the membrane- or fiber-forming processes. $^{23}$

$\chi$-parameter is empirically given by the following equation. ${ }^{15}$

$$
\chi=\chi_{0}\left(1+\sum_{j=1}^{n} p_{j} v_{p}^{j}\right)
$$

where

$$
\begin{aligned}
\chi_{0} & =\chi_{00}\left(1+k^{\prime} / X_{n}\right) \\
& =(a+b / T)\left\{1+\frac{k_{0}}{X_{n}}\left(1-\frac{\theta}{T}\right)\right\}
\end{aligned}
$$

Here $\chi_{00}$ is a parameter independent of $v_{p}$ and of $X_{i}$, and $k^{\prime}$ and $p_{j}$ are the molecular weight- and concentration-dependence parameters, respectively. $\theta$ is the Flory theta temperature, $T$ is Kelvin temperature, and $a, b$, and $k_{0}$ are coefficients independent of $X_{n}, v_{p}$, and $T$. Based on the Flory-Huggins theory, ${ }^{14}$ the chemical potential of the solvent $\Delta \mu_{0}$ and $X_{i}$-mer $\Delta \mu_{X_{i}}$ are expressed by eq 7 and 8 , respectively. ${ }^{15}$

$$
\begin{aligned}
& \Delta \mu_{0}= \tilde{R} T\left\{\ln \left(1-v_{p}\right)+\left(1-\frac{1}{X_{n}}\right) v_{p}\right. \\
&\left.+\chi_{00}\left(1+\frac{k^{\prime}}{X_{n}}\right)\left(1+\sum_{j=1}^{n} p_{j} v_{p}^{j}\right) v_{p}^{2}\right\} \\
& \Delta \mu_{X_{i}}=\tilde{R} T\left[\ln v_{X_{i}}-\left(X_{i}-1\right)+X_{i}\left(1-\frac{1}{X_{n}}\right) v_{p}\right. \\
&+X_{i}\left(1-v_{p}\right)^{2} \chi_{00}\left[\left(1+\frac{k^{\prime}}{X_{n}}\right)\right.
\end{aligned}
$$




$$
\begin{aligned}
& \times\left\{1+\sum_{j=1}^{n} \frac{p_{j}}{j+1}\left(\sum_{q=0}^{j}(q+1) v_{p}^{q}\right)\right\} \\
& +k^{\prime}\left(\frac{1}{X_{i}}-\frac{1}{X_{n}}\right)\left\{\frac{1}{1-v_{p}}\right. \\
& \left.\left.\left.+\sum_{j=1}^{n} \frac{p_{j}}{j+1}\left(\sum_{q=0}^{j} \frac{v_{p}^{q}}{1-v_{p}}\right)\right\}\right]\right] \\
& (i=1, \cdots, m)
\end{aligned}
$$

Here, $\tilde{R}$ is the gas constant. We assume that (a) the polymer and solvent are volumetrically additive, and (b) the densities of polymer and solvent are the same. Then, the $\Delta \mu_{0}$ and $\Delta \mu_{X_{i}}$ in eq 7 and 8 satisfy the Gibbs-Duhem relation. The well-known Gibbs conditions for the two-phase equilibrium of a quasibinary solution at constant temperature and pressure are,

$$
\begin{aligned}
& \Delta \mu_{0(1)}=\Delta \mu_{0(2)} \\
& \Delta \mu_{X_{i}(1)}=\Delta \mu_{X_{i}(2)}(i=1, \cdots, m)
\end{aligned}
$$

Here, the suffixes (1) and (2) denote the polymerlean and -rich phases respectively. Combination of eq $7-10$ gives

$$
\begin{aligned}
\chi_{00}= & \left\{\ln \frac{1-v_{p(1)}}{1-v_{p(2)}}+\left(v_{p(1)}-v_{p(2)}\right)\right. \\
& \left.-\left(\frac{v_{p(1)}}{X_{n(1)}}-\frac{v_{p(2)}}{X_{n(2)}}\right)\right\} /\left[\left(v_{p(2)}^{2}-v_{p(1)}^{2}\right)\right. \\
& +k^{\prime}\left(\frac{v_{p(2)}^{2}}{X_{n(2)}}-\frac{v_{p(1)}^{2}}{X_{n(1)}}\right) \\
& +\sum_{j=1}^{n} p_{j}\left\{\left(v_{p(2)}^{j+2}-v_{p(1)}^{j+2}\right)\right. \\
& \left.\left.+k^{\prime}\left(\frac{v_{p(2)}^{j+2}}{X_{n(2)}}-\frac{v_{p(1)}^{j+2}}{X_{n(1)}}\right)\right\}\right]
\end{aligned}
$$

and

$$
\sigma_{i} \equiv \frac{1}{X_{i}} \ln \frac{v_{X_{i}(2)}}{v_{X_{i}(1)}}=\sigma_{0}+\sigma_{01} / X_{i}
$$

with

$$
\begin{aligned}
\sigma_{0}= & \left(v_{p(1)}-v_{p(2)}\right)-\left(\frac{v_{p(1)}}{X_{n(1)}}-\frac{v_{p(2)}}{X_{n(2)}}\right) \\
& -\chi_{00}\left[2\left(v_{p(1)}-v_{p(2)}\right)-\left(v_{p(1)}^{2}-v_{p(2)}^{2}\right)\right. \\
& +\sum_{j=1}^{n} p_{j}\left\{\frac{j+2}{j+1}\left(v_{p(1)}^{j+1}-v_{p(2)}^{j+1}\right)\right. \\
& \left.\left.-\left(v_{p(1)}^{j+2}-v_{p(2)}^{j+2}\right)\right\}\right]-\chi_{00} k^{\prime}\left[\left(\frac{v_{p(1)}}{X_{n(1)}}-\frac{v_{p(2)}}{X_{n(2)}}\right)\right. \\
& -\left(\frac{v_{p(1)}^{2}}{X_{n(1)}}-\frac{v_{p(2)}^{2}}{X_{n(2)}}\right)+\sum_{j=1}^{n} p_{j}\left\{\left(\frac{v_{p(1)}^{j+1}}{X_{n(1)}}\right.\right. \\
& \left.\left.\left.-\frac{v_{p(2)}^{j+1}}{X_{n(2)}}\right)-\left(\frac{v_{p(1)}^{j+2}}{X_{n(1)}}-\frac{v_{p(2)}^{j+2}}{X_{n(2)}}\right)\right\}\right]
\end{aligned}
$$

and

$$
\begin{aligned}
\sigma_{01}= & -k^{\prime} \chi_{00}\left\{\left(v_{p(1)}-v_{p(2)}\right)\right. \\
& \left.+\sum_{j=1}^{n} \frac{p_{j}}{j+1}\left(v_{p(1)}^{j+1}-v_{p(2)}^{j+1}\right)\right\}
\end{aligned}
$$

If $k^{\prime}$ and $p_{j}(j=1, \cdots, n)$ are known, $\chi_{00}, \sigma_{0}$, and $\sigma_{01}$ become functions of $v_{p(1)}, v_{p(2)}, X_{n(1)}$, and $X_{n(2)}$. According to definition, $v_{p(1)}, v_{p(2)}$, $X_{n(1)}$, and $X_{n(2)}$ are expressed in terms of $v_{X_{i}(1)}$ and $v_{X_{i}(2)}$ as follows.

$$
\begin{gathered}
v_{p(1)}=\sum_{i=1}^{m} v_{X_{i}(1)} \\
v_{p(2)}=\sum_{i=1}^{m} v_{X_{i}(2)} \\
X_{n(1)}=\sum_{i=1}^{m} v_{X_{i}(1)} /\left(\sum_{i=1}^{m} v_{X_{i}(1)} / X_{i}\right) \\
X_{n(2)}=\sum_{i=1}^{m} v_{X_{i}(2)} /\left(\sum_{i=1}^{m} v_{X_{i}(2)} / X_{i}\right)
\end{gathered}
$$

As expressed by eq $1-4, v_{X_{i}(1)}^{c p}$ and $v_{X_{i}(2)}^{c p}$ are determined by $\sigma_{i}^{c p}$ alone. Considering eq $11-16$, we can give $\chi_{00}, \sigma_{0}$, and $\sigma_{01}$ at the cloud point (referred to as $\chi_{00}^{c p}, \sigma_{0}^{c p}$, and $\sigma_{01}^{c p}$ ) as functions of assumed $\sigma_{0}$ and $\sigma_{01}$ (denoted as $\sigma_{0}^{a}$ and $\sigma_{01}^{a}$ ).

$$
\chi_{00}^{c p}=\chi_{00}^{c p}\left(\sigma_{0}^{a}, \sigma_{01}^{a}\right)
$$




$$
\begin{aligned}
& \sigma_{0}^{c p}=\sigma_{0}^{c p}\left(\sigma_{0}^{a}, \sigma_{01}^{a}\right) \\
& \sigma_{01}^{c p}=\sigma_{01}^{c p}\left(\sigma_{0}^{a}, \sigma_{01}^{a}\right)
\end{aligned}
$$

Here, solving following simultaneous equations,

$$
\begin{aligned}
& \sigma_{0}^{c p}\left(\sigma_{0}^{a}, \sigma_{01}^{a}\right)-\sigma_{0}^{a}=0 \\
& \sigma_{01}^{c p}\left(\sigma_{0}^{a}, \sigma_{01}^{a}\right)-\sigma_{01}^{a}=0
\end{aligned}
$$

we can obtain true $\sigma_{0}^{c p}$ and $\sigma_{01}^{c p}$. Substituting $\sigma_{0}^{c p}$ and $\sigma_{01}^{c p}$ into eq 17, we can evaluate $\chi_{00}^{c p}$. $\chi_{0}^{c p}$ can also be calculated by substituting $\chi_{00}^{c p}$ into eq 6 . Of course, utilizing eq $1-4,15 \mathrm{a}$, and $\mathrm{b}$, we can calculate $v_{X_{i}(1)}, v_{X_{i}(2)}, v_{p(1)}$, and $v_{p(2)}$. This procedure is almost the same as that of CPC calculation, reported in a previous paper. ${ }^{15}$

Koningsveld et al. ${ }^{9}$ have carried out only the calculation of phase-diagrams (in other words, composition) of cloud point curve and shadow curve (incipient phase curve). In their study, CPC was only indirectly calculated as a cross point of $v_{p}^{0}$ constant line and limiting tie-line of coexisting curve at a few and isolated $v_{p}^{0}$.

In this article, (i) a direct method without any serious assumptions was employed and (ii) not only the phase-diagram, but also the partition coefficient $\sigma$, and the molecular characteristics $\left(v_{X_{i}}^{c p}, X_{w}^{c p}, \quad\left(X_{w}\right)\right.$ $\left.X_{n}\right)^{c p}$, and the standard deviation $\sigma^{\prime c p}$ $\left(\equiv\left\{X_{w}^{c p}\left(X_{z}^{c p}-X_{n}^{c p}\right)\right\}^{1 / 2}\right) ; X_{w}^{c p}$ and $X_{z}^{c p}$ are the weight- and $z$-average of $X_{i}$, respectively) were calculated. CPC can be estimated from $\chi_{0}^{c p}$, and the shadow curve from $v_{p}^{c p}$. Both $v_{p}$ and $\chi_{00}$ at critical solution point $\left(v_{p}^{c}\right.$ and $\left.\chi_{00}^{c}\right)$ can be calculated by solving simultaneous equations 22 (spinodal condition) and 23 (neutral equilibrium condition): ${ }^{15}$

$$
\begin{gathered}
\frac{1}{X_{w} v_{p}}+\frac{1}{1-v_{p}}-\chi_{00}\left\{1+k^{\prime}\left(1+\frac{1}{X_{n}}-\frac{X_{w}}{X_{n}}\right)\right\} \\
\times\left\{2+\sum_{j=1}^{n} p_{j}(j+2) v_{p}^{j}\right\}=0
\end{gathered}
$$

$$
\begin{aligned}
& \frac{1}{\left(1-v_{p}\right)^{2}}-\frac{X_{z}}{\left(X_{w} v_{p}\right)^{2}} \\
& -\chi_{00}\left\{1+k^{\prime}\left(1+\frac{1}{X_{n}}-\frac{X_{w}}{X_{n}}\right)\right\} \\
& \times\left\{\sum_{j=1}^{n} p_{j} j(j+2) v_{p}^{j-1}\right\}=0
\end{aligned}
$$

\section{COMPUTER SIMULATION}

Following parameters were calculated under the same conditions as those of actual CPC experiments on polystyrene/cyclohexane system, reported in the previous paper. ${ }^{15}$ The original polymer was assumed to have the Schulz-Zimm type distribution,

$$
g_{0}\left(X_{i}\right)=\frac{y^{h+1}}{\Gamma(h+1)} X_{i}^{h} \exp \left(-y X_{i}\right)
$$

with

$$
y=h / X_{n}^{0}
$$

and

$$
h=1 /\left(X_{w}^{0} / X_{n}^{0}-1\right)
$$

with $X_{w}^{0}=2117$ and $X_{w}^{0} / X_{n}^{0}=2.8$. The 1 st and 2nd order concentration dependent coefficients $p_{1}$ and $p_{2}$ and molecular-weight dependent coefficient $k_{0}$ werè taken as $0.643,0.200$, and 0 , respectively. $v_{p}^{c}$ and $\chi_{0}^{c}$ for the solution were 0.0766 and 0.5066 , respectively. In the case of $v_{p}^{0}<v_{p}^{c}$, two phase separation characteristics were calculated under the same conditions to compare $v_{p}^{c p}, \sigma^{c p}, \chi_{0}^{c p}, X_{w}^{c p},\left(X_{w} / X_{n}\right)^{c p}$, and $\sigma^{\prime c p}$ with $v_{p}, \sigma, \chi_{0}, X_{w}, X_{w} / X_{n}$ and $\sigma^{\prime}$ at two phase equilibrium $\left(\rho_{p}>0\right)$, respectively. Moreover, under the conditions of $X_{w}^{0} / X_{n}^{0}=$ $1.1-4, X_{w}^{0}=3 \times 10^{2}-5 \times 10^{3}, p_{1}=0-0.8$, and $p_{2}=0-0.4$, computer experiments on the cloud particle were carried out to study the effects of $X_{w}^{0} / X_{n}^{0}, X_{w}^{0}, p_{1}$, and $p_{2}$ on the cloud particle characteristics.

The computer experiments were carried out using FACOM M360. 


\section{RESULTS AND DISCUSSION}

Figure 1 shows the effect of $v_{p}^{0}$ on $v_{p(1)}^{c p}, v_{p(2)}^{c p}$, $\sigma^{c p}$, and $\chi_{0}^{c p}$. A chain line in Figure la) is a line of $v_{p(1)}^{c p}$ or $v_{p(2)}^{c p}=v_{p}^{0}$. The concentration of cloud particles $v_{p}^{c p}$ is $v_{p(2)}^{c p}$ for $v_{p}^{0}<v_{p}^{c}$ and $v_{p(1)}^{c p}$ for $v_{p}^{0}>v_{p}^{c}$.

The polymer volume fraction of the cloud particles $v_{p}^{c p}$ decreases gradually with an increase in $v_{p}^{0}$. At CSP, the following relation holds (filled circle):

$$
v_{p}^{c}=v_{p(1)}^{c p}=v_{p(2)}^{c p}
$$

$\sigma^{c p}$ decreases first with an increase in $v_{p}^{0}$ and shows a rather sharp minimum at CSP. At the limit of infinite dilution i.e., $\left(v_{p}^{0} \rightarrow 0\right)$, $v_{p(1)}^{c p}$ becomes zero, and accordingly $\sigma^{c p}$ becomes infinite (see, eq 12). Note that $\sigma^{c p} v s$. $v_{p}^{0}$ curve is continuous except for singular point $v_{p}^{0}=v_{p}^{c}$ (unfilled circle).

Mathematically, the following relations hold:

$$
\begin{gathered}
\lim _{v_{p}^{0} \rightarrow v_{p}^{c-}} \sigma^{c p}\left(\equiv \sigma_{c s p-}^{c p}\right)=\lim _{v_{p}^{0} \rightarrow v_{p}^{c+}} \sigma^{c p}\left(\equiv \sigma_{c s p+}^{c p}\right)=0 \\
\lim _{v_{p}^{0} \rightarrow v_{p}^{c}-} \frac{\partial \sigma^{c p}}{\partial v_{p}^{0}} \neq \lim _{v_{p}^{0} \rightarrow v_{p}^{c}+} \frac{\partial \sigma^{c p}}{\partial v_{p}^{0}}
\end{gathered}
$$

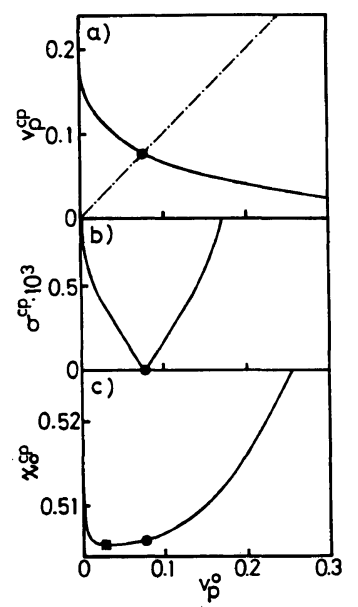

Figure 1. The polymer volume fraction of cloud particle $v_{p}^{c p}$, the partition coefficient and $\chi_{0}$-parameter at cloud point, $\sigma^{c p}$ and $\chi_{0}^{c p}$, plotted as a function of $v_{p}^{0}$ : Original polymer, Schulz-Zimm type distribution $\left(X_{w}^{0}=\right.$ 2117, $\left.X_{w}^{0} / X_{n}^{0}=2.8\right) ; p_{1}=0.643, p_{2}=0.200, k_{0}=0$.

$$
\sigma^{c p}=0
$$

(when $v_{p}^{0}=v_{p}^{c}$; denoted as filled circle)

Koningsveld and Staverman ${ }^{9}$ estimated, by applying an indirect method for Rehage et al.'s data $^{24}$ on polystyrene/cyclohexane system, a continuous shadow curve including CSP (see Figure 8 of ref 9). They draw a line passing through several points near and apart from CSP. Note that their indirect method becomes very inaccurate near CSP. We can draw continuous shadow line as shown in Figure 2 (broken line).

At the limit of $v_{p}^{0} \rightarrow 0, \chi_{0}^{c p}$ approaches to infinity. With an increase in $v_{p}^{0}, \chi_{0}^{c p}$ decreases and then increases after passing minimum at threshold CP $\left(v_{p}^{0}=v_{p}{ }^{t c p}\right) . \chi_{0}$ vs. $v_{p}^{0}$ curve is continuous even at CSP (Figure 1c)).

Figure 3 shows the distribution of volume fraction of the polymer partitioned in the cloud particle, $v_{X_{i}}^{c p}$ (referred to as $v^{c p}\left(X_{i}\right)$ ). For $v_{p}^{0}<v_{p}^{c} \quad v^{c p}\left(X_{i}\right)=v_{(2)}^{c p} \quad\left(X_{i}\right)$ and for $v_{p}^{0}>v_{p}^{c}$ $v^{c p}\left(X_{i}\right)=v_{(1)}^{c p}\left(X_{i}\right)$ hold. As $v_{p}^{0}$ becomes higher in the range of $v_{p}^{0}<0.1, v^{c p}\left(X_{i}\right)$ becomes apparently sharp and the content of higher $X_{i}$ component in cloud particle decreases. At $v_{p}^{0}=v_{p}^{c}$ (in this case, 0.0766) $v^{c p}\left(X_{i}\right)$ coincides with volume fraction of $X_{i}$ component in the original polymer $v_{0}\left(X_{i}\right)$. The peak height

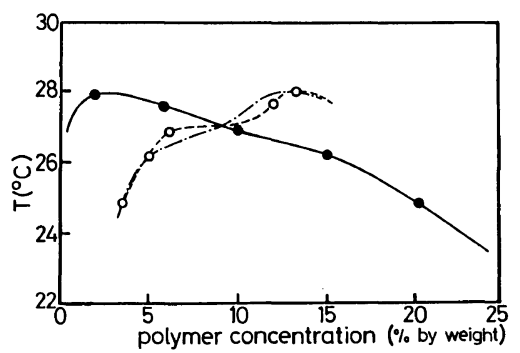

Figure 2. Cloud point curve and shadow curve of polystyrene/cyclohexane system: cloud point extrapolated using an indirect method by KoningsveldStaverman ${ }^{9}$ from Rehage et al.' $\mathrm{s}^{24}$ coexisting curve; $\bigcirc$, shadow point extrapolated using an indirect method by Koningsveld-Staverman ${ }^{9}$ from Rehage et al.' $\mathrm{s}^{24}$ coexisting curve. - cloud point curve estimated by Koningsveld and Staverman; -----, shadow curve estimated by Koningsveld and Staverman; ----, shadow curve estimated in this paper. 
of $v^{c p}\left(X_{i}\right)$ attains maximum at $v_{p}^{0}=0.1$, then decreases for a further increase in $v_{p}^{0}$, and $v^{c p}\left(X_{i}\right)$ approaches again $v_{0}\left(X_{i}\right)$ at limit of $v_{p}^{0} \rightarrow 1$.

Figure 4 shows $X_{w}^{c p},\left(X_{w} / X_{n}\right)^{c p}$, and $\sigma^{c p}$ as functions of $v_{p}^{0}$. At the limit of $v_{p}^{0} \rightarrow 0, X_{w}^{c p}$ becomes infinite and $\left(X_{w} / X_{n}\right)^{c p}$ and $\sigma^{\prime c p}$ approach to unity and zero, respectively. With an increase in $v_{p}^{0} X_{w}^{c p}$ decreases first very remarkably down to the value smaller than $X_{w}$ of the original polymer, $X_{w}^{0}$ (filled mark in the figure) and then increases very gradually after passing through minimum (unfilled mark), approaching to $X_{w}^{0}$ again at $v_{p}^{0} \rightarrow 1$. With an increase in $v_{p}^{0},\left(X_{w} / X_{n}\right)^{c p}$ increases rapidly from unity (at $\left.v_{p}^{0}=0\right)$ and shows a maximum at a specific $v_{p}^{0}$ (in this case, $=0.045$; denoted as unfilled rectangle) and then slowly decreases. At CSP (i.e., $\left.v_{p}^{0}=v_{p}^{c}\right)\left(X_{w} / X_{n}\right)^{c p}$ coincides with $X_{w}^{0} / X_{n}^{0}$ and with a further increase in $v_{p}^{0}$ beyond $v_{p}^{c}$ $\left(X_{w} / X_{n}\right)^{c p}$ attains a minimum and approaches $X_{\mathrm{w}}^{0} / X_{n}^{0}$ again at $v_{p}^{0} \rightarrow 1$. It should be noted that $\left(X_{w} / X_{n}\right)^{c p}$ coincides with $X_{\mathrm{w}}^{0} / X_{n}^{0}$ at three different $v_{p}^{0}$. Among them, at the lowest $v_{p}^{0} X_{w}^{c p}$ is very larger than $X_{w}^{0}$ and of course

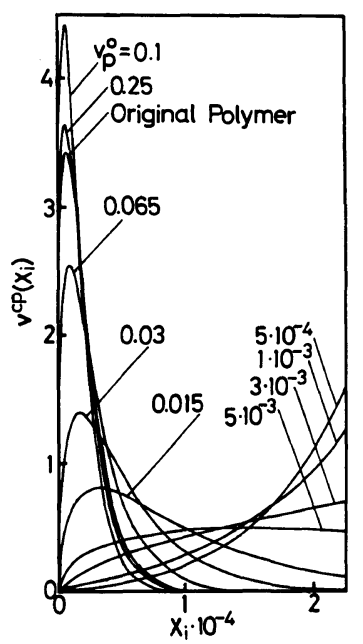

Figure 3. The distribution function of polymer volume fraction of cloud particle $v^{c p}\left(X_{i}\right)$. Original polymer, Schulz-Zimm type distribution $\left(X_{w}^{0}=2117, X_{w}^{0} / X_{n}^{0}=\right.$ 2.8); $p_{1}=0.643, p_{2}=0.200, k_{0}=0$. Number on curve denotes $v_{p}^{0}$. $v^{c p}\left(X_{i}\right)$ does not coincides with $v_{0}\left(X_{i}\right)$. Therefore, the polymer having the same $v^{c p}\left(X_{i}\right)$ as the original polymer is expected to appear at both $v_{p}^{0}=v_{p}^{c}$ and $v_{p}^{0} \rightarrow 1$, in which no phase separation occurs. $\sigma^{\prime c p}$ reveals minimum at specific $v_{p}^{0}$ between $v_{p}^{c}$ and 1. The specific $v_{p}^{0}$, giving minimum $X_{w}^{c p},\left(X_{w} / X_{n}\right)^{c p}$, and $\sigma^{\prime c p}$ is the same $(\simeq 0.1$, denoted as unfilled circle), and referred to as $v_{p \text {, min }}^{0}$. Theoretically, the following three equations are satisfied simultaneously at $v_{p}^{0}=v_{p, \min }^{0}$.

$$
\begin{gathered}
\frac{\partial X_{w}^{c p}}{\partial v_{p}^{0}}=0 \\
\frac{\partial\left(X_{w} / X_{n}\right)^{c p}}{\partial v_{p}^{0}}=0 \\
\frac{\partial \sigma^{\prime c p}}{\partial v_{p}^{0}}=0
\end{gathered}
$$

Figures $5 \mathrm{a}$ and $\mathrm{b}$ show $v_{p(2)}^{c p}\left(\right.$ or $\left.v_{p(1)}^{c p}\right) v s . v_{p}^{0}$

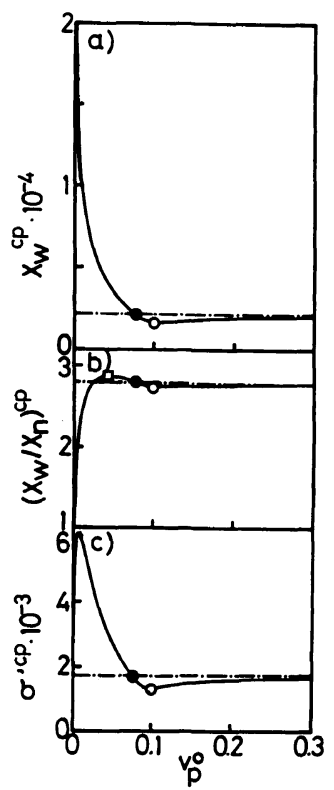

Figure 4. Plot of the weight-average, $X_{i}$, the ratio $X_{w} / X_{n}$ and the standard deviation of the polymer in cloud particle, $X_{w}^{c p},\left(X_{w} / X_{n}\right)^{c p}$, and $\sigma^{c p}$, as a function of $v_{p}^{0}$. Original polymer, Schulz-Zimm type distribution $\left(X_{w}^{0}=2117, X_{w}^{0} / X_{n}^{0}=2.8\right) ; p_{1}=0.643, p_{2}=0.200, k_{0}=0$. Filled and unfilled circles are correspond to $v_{p}^{c}$ and $v_{p, \min }^{0}$, respectively. 


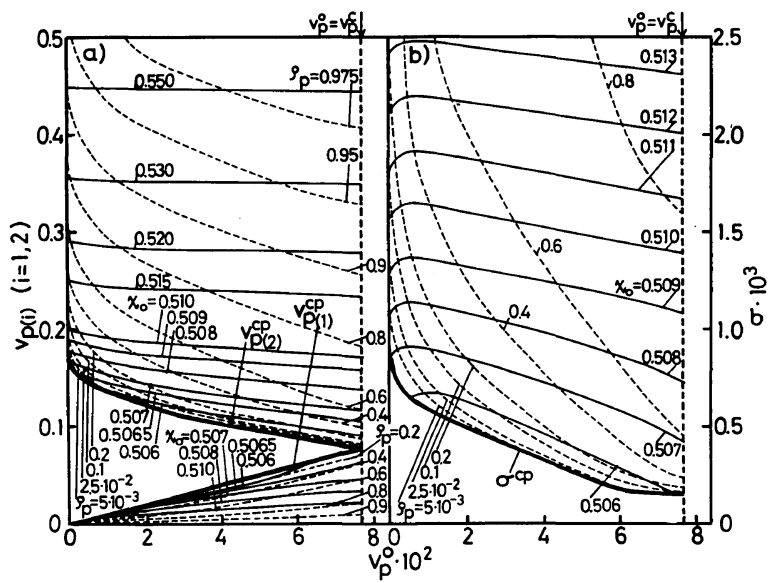

Figure 5. a) Comparison of the polymer volume fraction at cloud point $v_{p(i)}^{c p} v s . v_{p}^{0}$ curve (broad solid line) with the polymer volume fraction at constant $\rho_{p}$ (fine broken line; $\rho_{p}=5 \times 10^{-3}-0.975$ ). Suffix $i$ denotes the polymer-lean phase in the case of $i=1$ and the polymer-rich phase in the case of $i=2$. b) The comparison of the partition coefficient at cloud point $\sigma^{c p} v s . v_{p}^{0}$ curve (broad solid line) with the partition coefficient $\sigma v$ s. $v_{p}^{0}$ curve at constant $\rho_{p}$ (fine broken line; $\rho_{p}=5 \times 10^{-3}-0.8$ ). Fine solid lines are the contour lines of $\chi_{0}$. Original polymer, Schulz-Zimm type distribution $\left(X_{w}^{0}=2117, X_{w}^{0} / X_{n}^{0}=2.8\right) ; p_{1}=0.643$, $p_{2}=0.200, k_{0}=0$.

curve and $\sigma^{c p}$ vs. $v_{p}^{0}$ curve (shown as broad solid line). The figure includes $v_{p(2)}\left(\right.$ or $\left.v_{p(1)}\right)$ vs. $v_{p}^{0}$ curve and $\sigma$ vs. $v_{p}^{0}$ curve at constant $\rho_{p}$, ranging from 0.005 to 0.975 (shown as fine broken line), in the range of $v_{p}^{0}<v_{p}^{c}$. Figure 5 also includes the constant $\chi_{0}$ line (denoted as fine solid line). With a decrease in $\rho_{p}$ and $\chi_{0}$, $v_{p(2)}$ and $\sigma$ decrease and $v_{p(1)}$ increases. At the limit of $\rho_{p} \rightarrow 0, v_{p(1)} v s . v_{p}^{0}, v_{p(2)} v s . v_{p}^{0}$, and $\sigma v s$. $v_{p}^{0}$ curves converge into $v_{p(1)}^{c p}$. vs. $v_{p}^{0}$ (namely, $\left.v_{p(1)}^{c p}=v_{p}^{0}\right), v_{p(2)}^{c p} v s . v_{p}^{0}$, and $\sigma^{c p} v s$. $v_{p}^{0}$ curves, respectively. In the case of $k^{\prime}=0$, using eq 6 , we can get

$$
\chi_{0}=a+b / T
$$

and $v_{p(i)}(i=1,2)$ vs. $v_{p}^{0}$ and $\sigma$ vs. $v_{p}^{0}$ curves at constant $\chi_{0}$ value can be transformed into the corresponding curves at constant temperature (simply referred to as $T$ constant curve). Along the constant temperature curve with $\chi_{0} \geqq 0.507, \rho_{p}$ increases monotonically with an increase in $v_{p}^{0}$. In the case of $\chi_{0}=0.506$, with an increase in $v_{p}^{0} \rho_{p}$ increases first and shows a maximum $\left(\rho_{p}=0.2\right) . v_{p}^{0}$, which gives the smallest $\chi_{0}$, coincides with $v_{p}^{t c p}$.

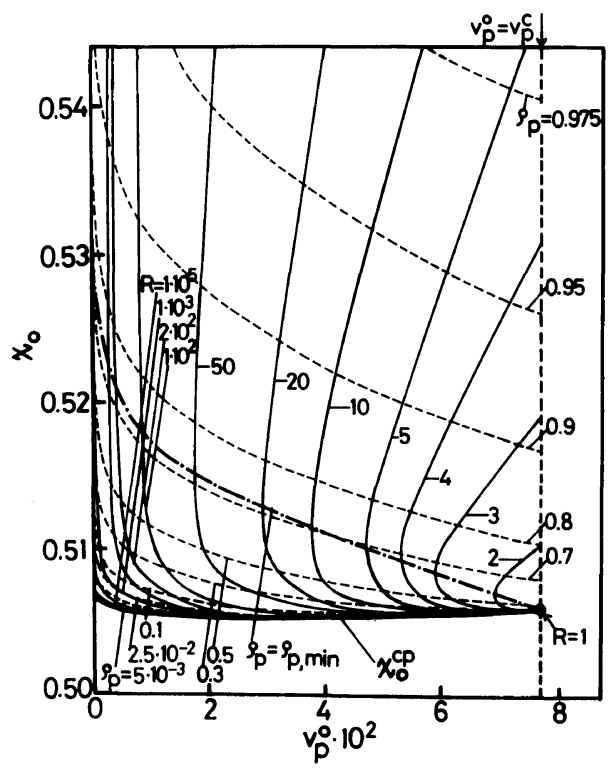

Figure 6. Comparison of $\chi_{0}$ at cloud point $\left(\chi_{0}^{c p}\right)$ vs. $v_{p}^{0}$ curve (broad solid line) with $\chi_{0} v s$. $v_{p}^{0}$ curve at constant $\rho_{p}$ (fine broken line; $\rho_{p}=5 \times 10^{3}-0.975$ ). Fine solid lines are the contour lines of phase volume ratio $R$. Original polymer, Schulz-Zimm type distribution $\left(X_{w}^{0}=2117\right.$, $\left.X_{w}^{0} / X_{n}^{0}=2.8\right) ; p_{1}=0.643, p_{2}=0.200, k_{0}=0$. Filled circle is the critical solution point. 


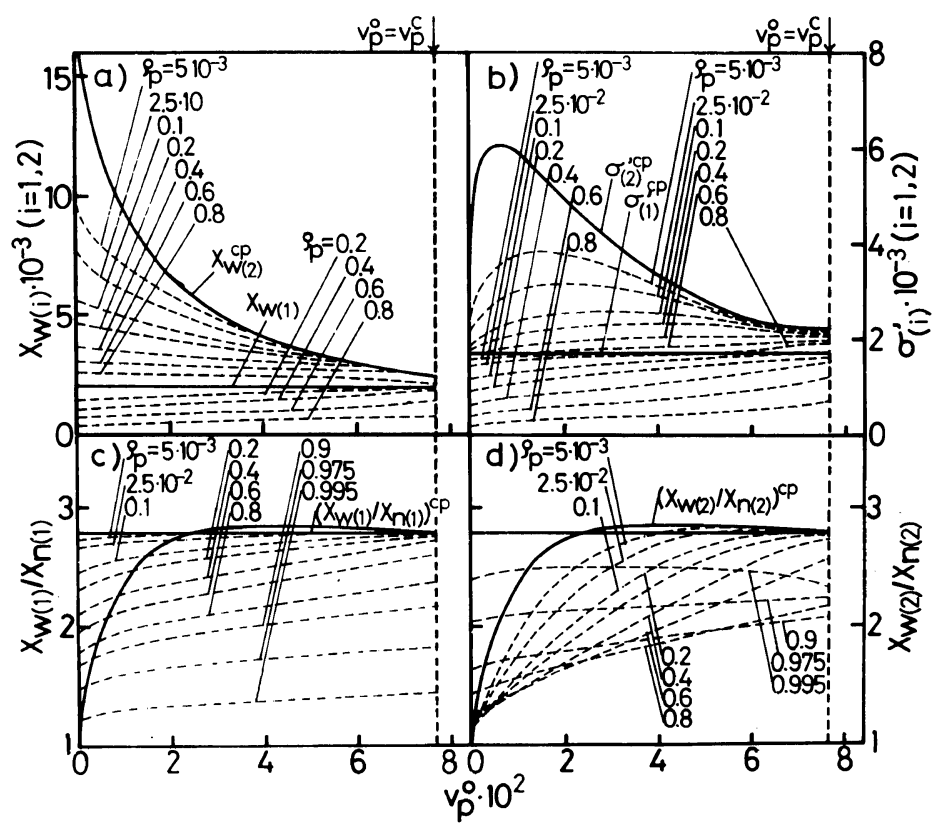

Figure 7. Comparison of a) $X_{w(i)}^{c p}$, b) $\sigma_{(i)}^{\prime c p}$, c) $\left(X_{w(1)} / X_{n(1)}\right)^{c p}$ and d) $\left(X_{w(2)} / X_{n(2)}\right)^{c p} v s . v_{p}^{0}$ curves (denoted as broad solid line) with a) $X_{w(i)}$, b) $\sigma_{(i)}^{\prime}$, c) $X_{w(1)} / X_{n(1)}$, and d) $X_{w(2)} / X_{n(2)} v s . v_{p}^{0}$ curves (denoted as fine broken line) at constant $\rho_{p}$ (ranging from $5 \times 10^{-3}$ to 0.995 ), respectively. Original polymer, Schulz-Zimm type distribution $\left(X_{w}^{0}=2117, X_{w}^{0} / X_{n}^{0}=2.8\right) ; p_{1}=0.643, p_{2}=0.200, k_{0}=0$. Suffix $i$ denotes the polymer-lean phase in the case of $i=1$ and the polymer-rich phase in the case of $i=2$.

A comparison of $\chi_{0}^{c p}$ vs. $v_{p}^{0}$ curve (denoted as broad solid line) with $\chi_{0}$ vs. $v_{p}^{0}$ curve at constant $\rho_{p}$ (denoted as fine broken line) is shown in Figure 6. CSP is denoted by filled circle. With a decrease in $\rho_{p}, \chi_{0}$ decreases and finally $\chi_{0} v s . v_{p}^{0}$ curve coincides with $\chi_{0}^{c p} v s$. $v_{p}^{0}$ curve at the limit of $\rho_{p} \rightarrow 0$. Constant $R$ line is denoted as fine solid line in the figure. Contour line of $R$ also coincides with $\chi_{0}^{c p} v s$. $v_{p}^{0}$ curve for $R \rightarrow \infty$ and converges into CSP for $R \rightarrow 1$. With an increase in $\chi_{0}, R$ decreases first and shows minimum $\left(\equiv R_{\min }\right) .{ }^{1-8}$ The relation between $R_{\min }$ and $v_{p}^{0}$ is denoted as chain line. $R$ near CP is large $\left(\sim 1 \times 10^{5}\right)$ for small $v_{p}^{0}$ and decreases with an increase in $v_{p}^{0}$. In contrast to this, $\rho_{p}$ near CP is small $(\sim 0.005)$ for small $v_{p}^{0}$ and becomes large $(\sim 0.3)$ for large $v_{p}^{0}$. In other words, for small $v_{p}^{0}$ small amount of polymer is partitioned in small polymerrich phase near $\mathrm{CP}$ and polymer volume concentration is high. For large $v_{p}^{0}$ large

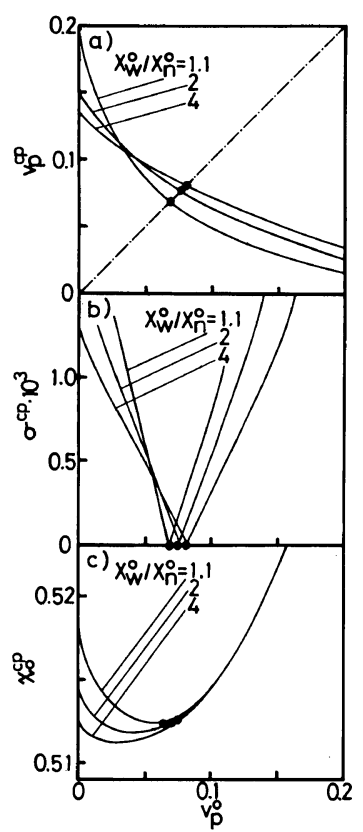

Figure 8. Effects of $X_{w}^{0} / X_{n}^{0}$ on $v_{p}^{c p}, \sigma^{c p}$, and $\chi_{0}^{c p} v s . v_{p}^{0}$ curves. Original polymer, Schulz-Zimm type distribution $\left(X_{w}^{0}=2000\right) ; p_{1}=0.6, p_{2}=0, k_{2}=0$. 
amount of polymer is partitioned in large polymer-rich phase near $\mathrm{CP}$, resulting in low polymer concentration.

Figures 7a, b, c, and d show $X_{w(2)}^{c p}\left(\right.$ or $\left.X_{w(1)}^{c p}\right)$ vs. $v_{p}^{0}, \sigma_{(2)}^{\prime c p}\left(\begin{array}{lllll}\text { or } & \sigma_{(1)}^{\prime c p}\end{array}\right)$ vs. $v_{p}^{0},\left(X_{w(1)} / X_{n(1)}\right)^{c p}$ vs. $v_{p}^{0}$, and $\left(X_{w(2)} / X_{n(2)}\right)^{c p}$ vs. $v_{p}^{0}$ curves (shown as broad solid line), respectively. The figure also shows $X_{w(2)}\left(\right.$ or $\left.X_{w(1)}\right) v s . v_{p}^{0}, \sigma_{(2)}^{\prime}\left(\right.$ or $\left.\sigma_{(1)}^{\prime}\right)$ vs. $v_{p}^{0}, X_{w(1)} / X_{n(1)} v s . v_{p}^{0}$, and $X_{w(2)} / X_{n(2)} v s . v_{p}^{0}$ curves (shown as fine broken line) with constant $\rho_{p}\left(\rho_{p}=0.005-0.995\right)$, in the range of $v_{p}^{0}<v_{p}^{c}$. At the limit of $\rho_{p} \rightarrow 0$, the molecular characteristics of two-phase equilibrium coincides excellently with the molecular characteristics of the cloud particle. As stated

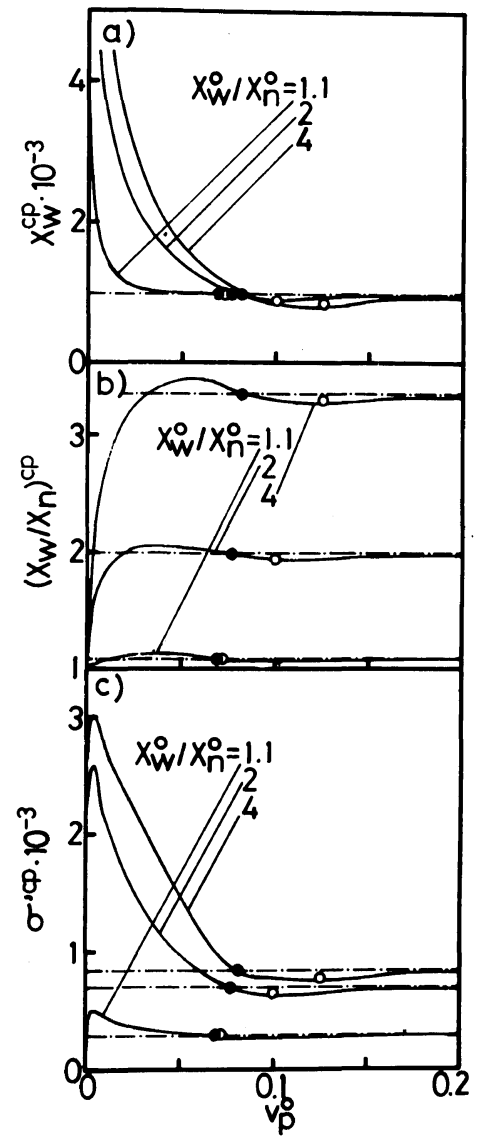

Figure 9. Effects of $X_{w}^{0} / X_{n}^{0}$ on $X_{w}^{c p},\left(X_{w} / X_{n}\right)^{c p}$, and $\sigma^{i c p}$ $v s . v_{p}^{0}$ curves. Filled and unfilled circles correspond to $v_{p}^{c}$ and $v_{p, \min }^{0}$, respectively. Original polymer, Schulz-Zimm type distribution $\left(X_{w}^{0}=2000\right) ; p_{1}=0.6, p_{2}=0, k_{0}=0$. above, the concept of cloud particle is rather hypothetical, but all the characteristics of cloud particle coincides with those of polymer in a polymer-rich phase in the case of $\rho_{p} \rightarrow 0$ and is expected to control the characteristics of primary particle, observed by Kamide and Manabe. $^{23}$

Figure 8 shows the effect of $X_{w}^{0} / X_{n}^{0}$ on the $v_{p}^{c p}, \sigma^{c p}$, and $\chi_{0}^{c p}$, as the functions of $v_{p}^{0} \cdot v_{p}^{c p}$ is strongly influenced by $\sigma^{c p}$, which decreases with an increase in $X_{w}^{0} / X_{n}^{0}$. With an increase in $\sigma^{c p}$ (namely, with an increase in $X_{w}^{0} / X_{n}^{0}$ ), $v_{p}^{c p}$ decrease for $v_{p}^{0}<v_{p}^{c}$, and $v_{p}^{c p}$ increase for $v_{p}^{0}>v_{p}^{c}$ (see, eq 2 and 3 ). Though $\chi_{0}^{c p}$ decreases with an increase in $X_{w}^{0} / X_{n}^{0}$ for $v_{p}^{0}<v_{p}^{c}, \chi_{0}^{c}$ is not significantly affected by $X_{w}^{0} / X_{n}^{0}$ for $v_{p}^{c}>$ $v_{p}^{c}$.

The effect of $X_{w}^{0} / X_{n}^{0}$ on $X_{w}^{c p},\left(X_{w} / X_{n}\right)^{c p}$, and $\sigma^{c p}$ is shown as the function of $v_{p}^{0}$ in Figure 9. As $X_{w}^{0} / X_{n}^{0}$ increases, $X_{w}^{c p}$ decreases in the case of $v_{p}^{0}<v_{p}^{c}$ and increases in the case of $v_{p}^{0}>v_{p}^{c} . v_{p, \min }^{0}$ increases with an increase in $X_{w}^{0} / X_{n}^{0}$. For $v_{p}^{0}<v_{p}^{c}\left(X_{w} / X_{n}\right)^{c p}$ as well as $\sigma^{\prime c p}$

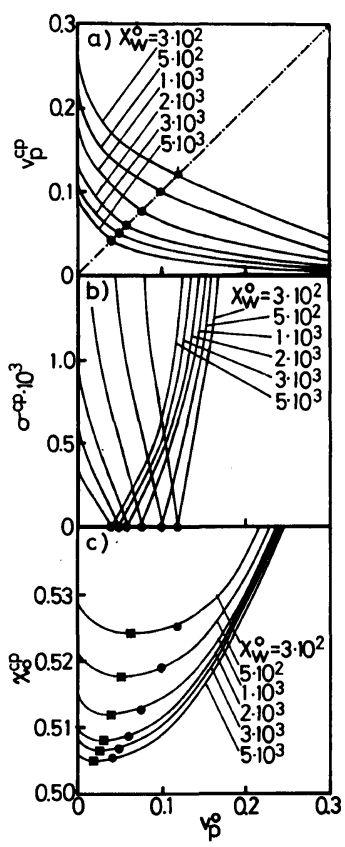

Figure 10. Effects of $X_{w}^{0}$ on $v_{p}^{c p}, \sigma^{c p}$, and $\chi_{0}^{c p} v s . v_{p}^{0}$ curves. Original polymer Schulz-Zimm type distribution $\left(X_{w}^{0} / X_{n}^{0}=0\right) ; p_{1}=0.6, p_{2}=0, k_{2}=0$. 
are strongly influenced by $X_{w}^{0} / X_{n}^{0}$.

Figure 10 shows the effect of $X_{w}^{0}$ on $v_{p}^{c p}, \sigma^{c p}$, and $X_{0}^{c p}$ vs. $v_{p}^{0}$ curves. With an increase in $X_{w}^{0}$, $\sigma^{c p}$ decreases for $v_{p}^{0}<v_{p}^{c}$ and increases for $v_{p}^{0}>v_{p}^{c}$. A change in $\sigma^{c p}$ with $X_{w}^{0}$ is the main cause of the decrease in $v_{p}^{c p}$ with an increase in $X_{w}^{0}$. As $X_{w}^{0}$ increases, $\chi_{0}^{c p}$ decreases. ${ }^{15}$

Figure 11 shows the effects of $X_{w}^{0}$ on $X_{w}^{c p}$, $\left(X_{w} / X_{n}\right)^{c p}$, and $\sigma^{\prime c p}$ vs. $v_{p}^{0}$ curves. $v_{p, \min }^{0}$ (shown as unfilled circle in the figure) decreases with an increase in $X_{w}^{0}$. The decrease in

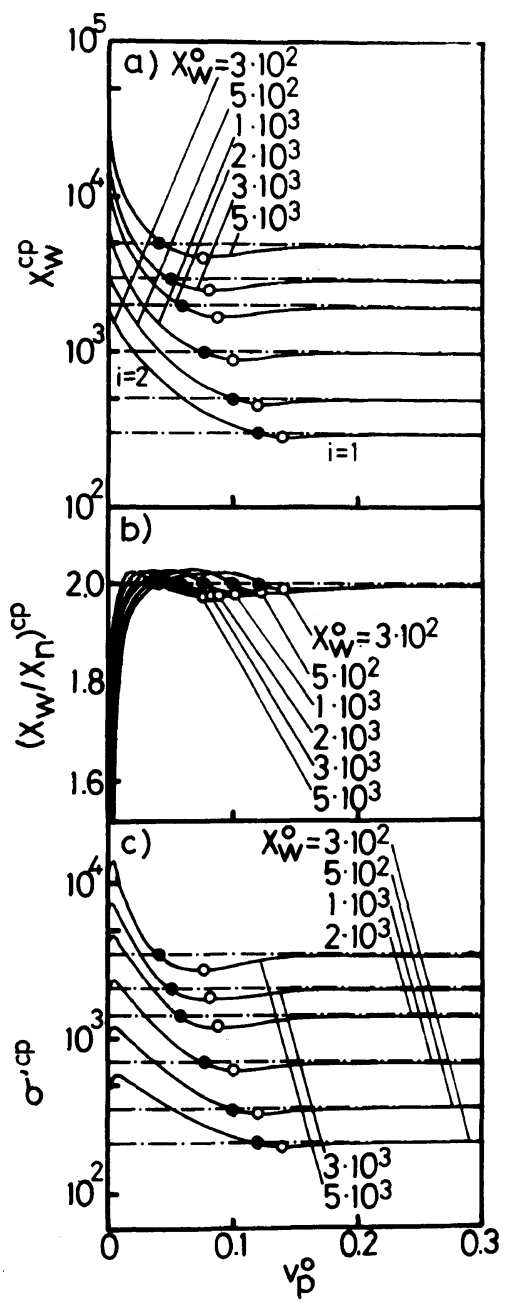

Figure 11. Effects of $X_{w}^{0}$ on $X_{w}^{c p},\left(X_{w} / X_{n}\right)^{c p}$, and $\sigma^{c p} v s$. $v_{p}^{0}$ curves. Filled and unfilled circles correspond to $v_{p}^{c}$ and $v_{p, \min }^{0}$, respectively. Original polymer, Schulz-Zimm type distribution $\left(X_{w}^{0} / X_{n}^{0}=2\right) ; p_{1}=0.6, p_{2}=0, k_{0}=0$. $v_{p}^{c}$ (denoted as filled circle) with an increase in $X_{w}^{0}$ seems to demand a decrease of $v_{p, \min }^{0}$. As $X_{w}^{0}$ increases, $\left(X_{w} / X_{n}\right)^{c p}$ increases for $v_{p}^{0}<v_{p}^{c}$ and decreases for $v_{p}^{0}>v_{p}^{c}$.

Figure 12 shows the effect of $p_{1}$ on $v_{p}^{c p}, \sigma^{c p}$, and $\chi_{0}^{c p}$ vs. $v_{p}^{0}$ curves. With an increase in $p_{1}, \sigma^{c p}$ increases in the range $v_{p}^{0}<v_{p}^{c}$ and decreases in the range $v_{p}^{0}>v_{p}^{c}$. The $p_{1}$ dependence of $\sigma^{c p}$ brings about an increase in $v_{p}^{c p}$ with an increase in $p_{1} \cdot \chi_{0}^{c p}$ decreases with an increase in $p_{1} . p_{1}$ has a strong effect on $v_{p}^{c}$, $v_{p}^{c p}, \sigma^{c p}$, and $\chi_{0}^{c p}$.

Figure 13 shows the effect of $p_{1}$ on $X_{w}^{c p}$, $\left(X_{w} / X_{n}\right)^{c p}$, and $\sigma^{\prime c p}$ as the functions of $v_{p}^{0}$. As $p_{1}$ increases, $X_{w}^{c p}$ increases and for $v_{p}^{0}>v_{p}^{c}$ $\left(X_{w} / X_{n}\right)^{c p}$ decreases. $v_{p, \text { min }}^{0}$ (unfilled circle) increases substantially with an increase in $p_{1}$. In the case of $v_{p}^{0}>v_{p, \text { min }}^{0}$ the molecular characteristics are almost independent of $p_{1}$.

Figures 14 and 15 show the effect of $p_{2}$ on

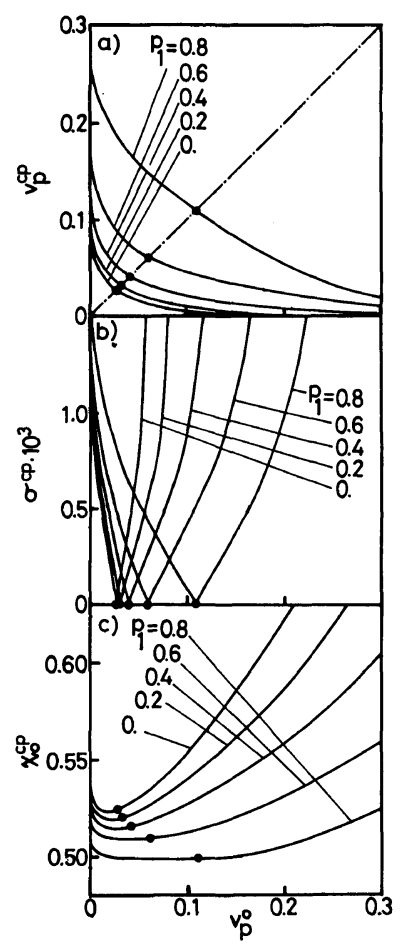

Figure 12. Effects of $p_{1}$ on $v_{p}^{c p}, \sigma^{c p}$, and $\chi_{0}^{c p} v s$. $v_{p}^{0}$ curves. Original polymer, Schulz-Zimm type distribution $\left(X_{w}^{0}=2000, X_{w}^{0} / X_{n}^{0}=2\right) ; p_{2}=0, k_{0}=0$. 


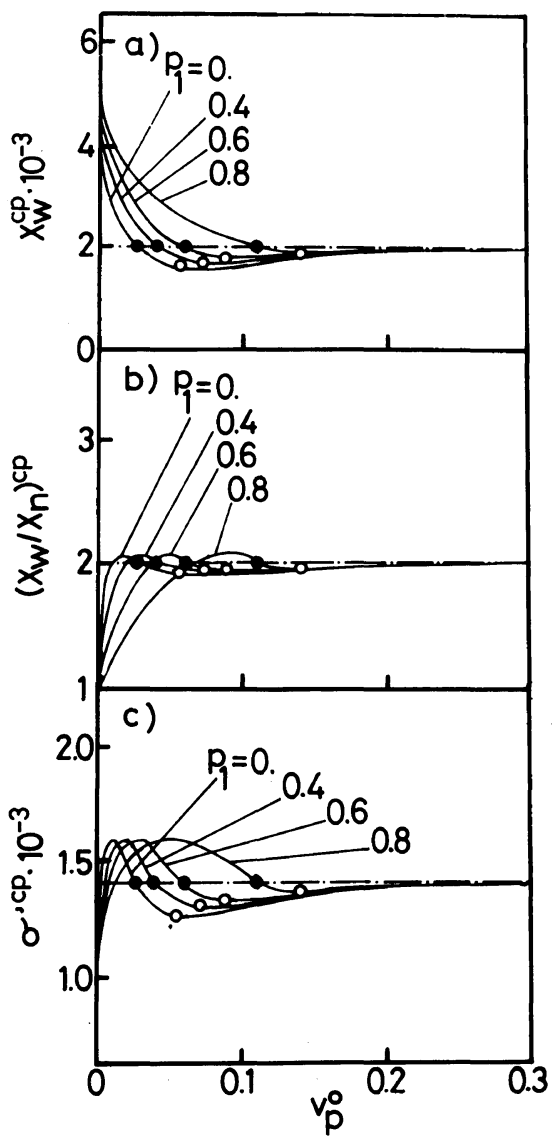

Figure 13. Effects of $p_{1}$ on $X_{w^{\prime}}^{c p},\left(X_{w} / X_{n}\right)^{c p}$, and $\sigma^{\prime c p} v s$. $v_{p}^{0}$ curves. Filled and unfilled circles correspond to $v_{p}^{c}$ and $v_{p, \min }^{0}$, respectively. Original polymer, Schulz-Zimm type distribution $\left(X_{w^{\prime}}^{0}=2000, X_{w^{0}}^{0} / X_{n}^{0}=2\right) ; p_{2}=0, k_{0}=0$.

both $\left(v_{p}^{c p}, \sigma^{c p}, \chi_{0}^{c p}\right)$ and $\left(X_{w}^{c p},\left(X_{w} / X_{n}\right)^{c p}\right.$ $\left.\sigma^{\prime c p}\right)$, respectively. $p_{2}$ has a smaller effect on characteristics of cloud particle than that of $p_{1}$. The effect of $p_{2}$ on cloud particle for $v_{p}^{0}>v_{p}^{c}$ is relatively larger than that for $v_{p}^{0}<$ $v_{p}^{c}$, because $p_{2}$ is the concentration dependent parameter of square $v_{p}$ term of $\chi$.

Acknowledgements. The author should like to express his most sincere gratitude to Dr. Kenji Kamide, Director of Fundamental Research Laboratory of Fibers and FiberForming Polymers, Asahi Chemical Industry Co., Ltd., for his guidance and stimulating.

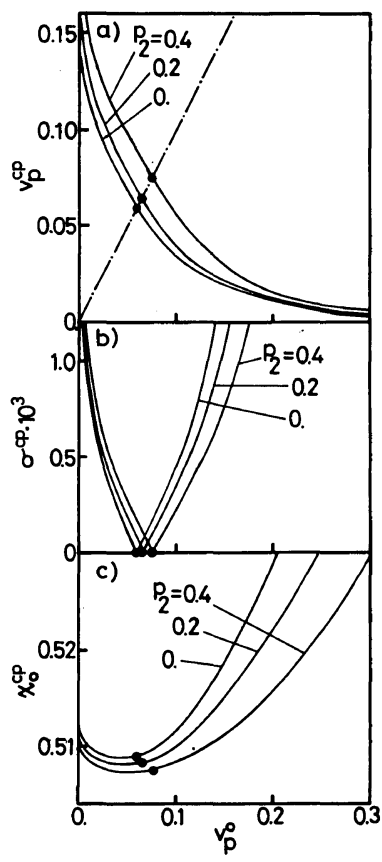

Figure 14. Effects of $p_{2}$ on $v_{p}^{c p}, \sigma^{c p}$, and $\chi_{0}^{c p} v s$. $v_{p}^{0}$ curves. Original polymer, Schulz-Zimm type distribution $\left(X_{w}^{0}=2000, X_{w}^{0} / X_{n}^{0}=2\right) ; p_{1}=0.6, k_{0}=0$.

comments.

\section{REFERENCES}

1. see, for example K. Kamide, in "Fractionation of Synthetic Polymers," L. H. Tung, Ed., Marcel Dekker Inc., New York, N. Y., 1977, Chapter 2.

2. K. Kamide, Y. Miyazaki, and T. Abe, Polym. J., 9, 395 (1977).

3. I. Noda, H. Ishizawa, Y. Miyazaki, and K. Kamide, Polym. J., 12, 87 (1980).

4. K. Kamide, K. Sugamiya, T. Kawai, and Y. Miyazaki, Polym. J., 12, 67 (1980).

5. K. Kamide and Y. Miyazaki, Polym. J., 12, 205 (1980).

6. K. Kamide and Y. Miyazaki, Polym. J., 13, 325 (1981).

7. K. Kamide, Y. Miyazaki, and T. Abe, Br. Polym. J., 13, 168 (1981).

8. K. Kamide, T. Abe, and Y. Miyazaki, Polym. J., 14, 355 (1982).

9. R. Koningsveld and A. J. Staverman, Kolloid Z.-Z. Polym., 218, 114 (1967).

10. R. Koningsveld and A. J. Staverman, J. Polym. Sci., $A-2,6,305$ (1968).

11. R. Koningsveld and A. J. Staverman, J. Polym. Sci., 


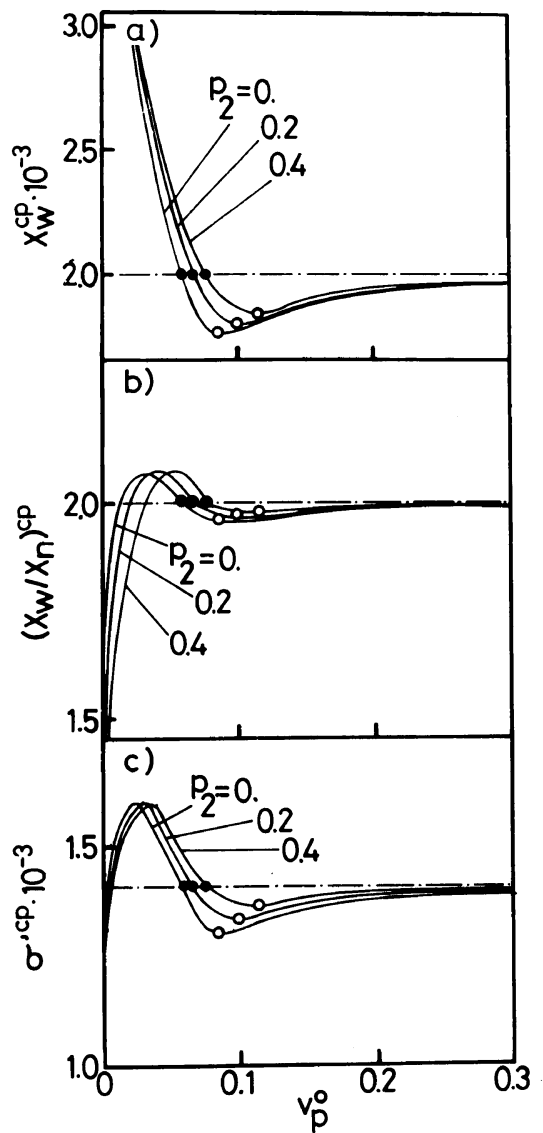

Figure 15. Effects of $p_{2}$ on $X_{w}^{c p},\left(X_{w} / X_{n}\right)^{c p}$, and $\sigma^{c p p} v s$. $v_{p}^{0}$ curves. Filled and unfilled circles correspond to $v_{p}^{c}$ and $v_{p \text {,min }}^{0}$, respectively. Original polymer, Schulz-Zimm type distribution $\left(X_{w}^{0}=2000, X_{w}^{0} / X_{n}^{0}=2\right) ; p_{1}=0.6, k_{0}=0$.
A-2, 6, 349 (1968).

12. M. Gordon, H. A. G. Chermin, and R. Koningsveld, Macromolecules, 2, 107 (1969).

13. R. Koningsveld, W. H. Stockmayer, J. W. Kennedy, and L. A. Kleintjens, Macromolecules, 7, 73 (1974).

14. P. J. Flory, "Principle of Polymer Chemistry," Cornell University Press, Ithaca, New York, 1953.

15. K. Kamide, S. Matsuda, T. Dobashi, and M. Kaneko, Polym. J., 16, 839 (1984).

16. K. Solc, Collect. Czech. Chem. Commun., 34, 992 (1969).

17. K. Šlc, Macromolecules, 3, 665 (1970).

18. K. Solc, Macromolecules, 8, 819 (1975).

19. K. Solc, Macromolecules, 10, 1101 (1977).

20. K. Solc, J. Polym. Sci., 20, 1947 (1982).

21. K. Solc, Macromolecules, 16, 236 (1983).

22. K. Solc, L. A. Kleintjens, and R. Koningsveld, Macromolecules, 17, 573 (1984).

23. K. Kamide and S. Manabe, ACS Symp. Ser., 269, 197 (1984).

24. G. Rehage, D. Moller, and O. Ernst, Makromol. Chem., 38, 232 (1965). 